\title{
A Blended Learning Model of Peer Support Training Program for Women with Breast Cancer
}

\author{
Hiroko Komatsu, Kaori Yagasaki, Wakako Osaka, \\ Hideko Yamauchi, Seigo Nakamura \\ Faculty of Nursing and Medical Care, Keio University \\ E-mail:hkomatsu@sfc.keio.ac.jp \\ Faculty of Nursing and Medical Care, Keio University \\ E-mail:yagasaki@sfc.keio.ac.jp \\ St. Luke's College of Nursing \\ E-mail: 12DN003p7Q-ts@slcn.ac.jp \\ St. Luke's International Hospital \\ E-mail: hideyama@luke.or.jp \\ School of Medicine, Showa University \\ E-mail: seigonak@gmail.com
}

\begin{abstract}
Objectives: Peer support is an important social support for cancer patients. A blended learning model of peer support training program in breast cancer was tested for feasibility.

Methods: We developed a peer support training program for women with breast cancer with a blended learning approach combining faceto-face methods with e-learning. After face-to-face orientation, a 20session e-learning program was provided on the Web followed by a one-day workshop including role playing. Log-files were recorded to assess the pattern of use in the e-learning program. The usability and acceptability were assessed by an evaluation questionnaire with a 5point Likert scale. Psychosocial outcomes were assessed over time (baseline, post-program, and 3-month follow-up) using repeated measured one-way analysis of variance.

Results: Twenty-eight women with breast cancer experience were recruited to the study. The rates of retention and assessment completion were high $(92.9 \%$ and $89.3 \%$, respectively). Participants favorably rated on the usability of the program. The mean time taken to complete the total of 20 sessions in the e-learning program was
\end{abstract}


18.69 days. There were no adverse psychosocial effects on the participants.

Conclusions: The blended learning model of the breast cancer peer support training program was feasible and well accepted by the peer support volunteers.

Keywords: feasibility study, breast cancer, peer group, distance learning, social behavior

\section{Introduction}

As a consequence of advances in early detection and treatment, surviving cancer is an established reality [1]. Thus, the psychological well-being and quality of life of cancer patients, particularly breast cancer patients, have been received a great deal of attention [2, 3]. Social support has psychosocial benefits [4, 5] and improves the quality of life of women with breast cancer [6]. Moreover, a recent study suggests that breast cancer patients with strong social support are less likely to develop recurrence and have better survival rates [7].

Peer support, referring to support offered to cancer patients by people who have relevant experiences [8], is different from therapeutic and educational programs provided by health professionals [9]. Peer support is increasingly recognized as an important social support to improve coping skills and provide hope to women with breast cancer [10]. Furthermore, peer support benefits not only patients but also peers [11].

The role of the leader is critical to the success of support groups [12, 13]. According to the previous studies, however, more than 50\% of the support groups have some difficulties, and leaders are at risk of stress and burnout $[12,13]$, in addition to potential vulnerability of peer support volunteers who have a history of cancer [9]. Although the training needs of leaders are often addressed [13, 14], there are few standardized peer support training programs.

Cancer is a public health issue in Japan, and drives national strategies. The Cancer Control Act was implemented in 2007 with the objectives of prevention and early diagnosis of cancer, standardization of treatment, and innovation in research. It also addresses the quality of life of cancer patients [15]. Given the growing number of cancer patients and survivors, the recently released interim evaluation report highlights the urgent need for peer support programs [16].

We developed a peer support training program for potential leaders of breast cancer support groups. It consists of a blended learning model, combining traditional face-to-face didactic methods with e-learning. The blended learning program compliments face-to-face sessions with e-learning modules [17]. Online learning is accessible and good for formative exercises, while face-to-face 
sessions are necessary for correct adaptation of a program, interaction, and reflection. A meta-analysis demonstrated that blended learning has a similar effectiveness to traditional methods [18].

The objective of this study was to assess the feasibility of the peer support training program. We also assessed psychosocial outcomes in the participants.

\section{Methods}

\subsection{Design}

This study used a one-group prospective measures design to evaluate the feasibility of a peer support training program. The study period was from May, 2010 to February, 2011. The study procedures were reviewed and approved by the Institutional Review Board at Keio University (Approval Number: No 169, 2010).

\subsection{Participants}

Women with breast cancer were approached for inclusion into the study through local peer support groups. Eligible participants were women with primary breast cancer who had been diagnosed at least 6 months previously, and who had attended more than two professionally-led breast support group meetings at St. Luke's College of Nursing and St. Luke's International Hospital, Tokyo, for a 3month period from May to July, 2010. Those who experienced recurrence were excluded. Twenty-eight women were recruited, and written informed consent was obtained.

\subsection{Procedure}

\subsubsection{Training Program}

We developed a one-month peer support training program using a blended learning approach, consisting of face-to-face sessions (an orientation of the program with an overview of the e-learning tools and a one-day workshop including role playing) and e-learning (self-learning and online collaboration) (Fig. 1). At the orientation, the participants shared their experiences with cancer, and motivations for participating in the peer support training program. 


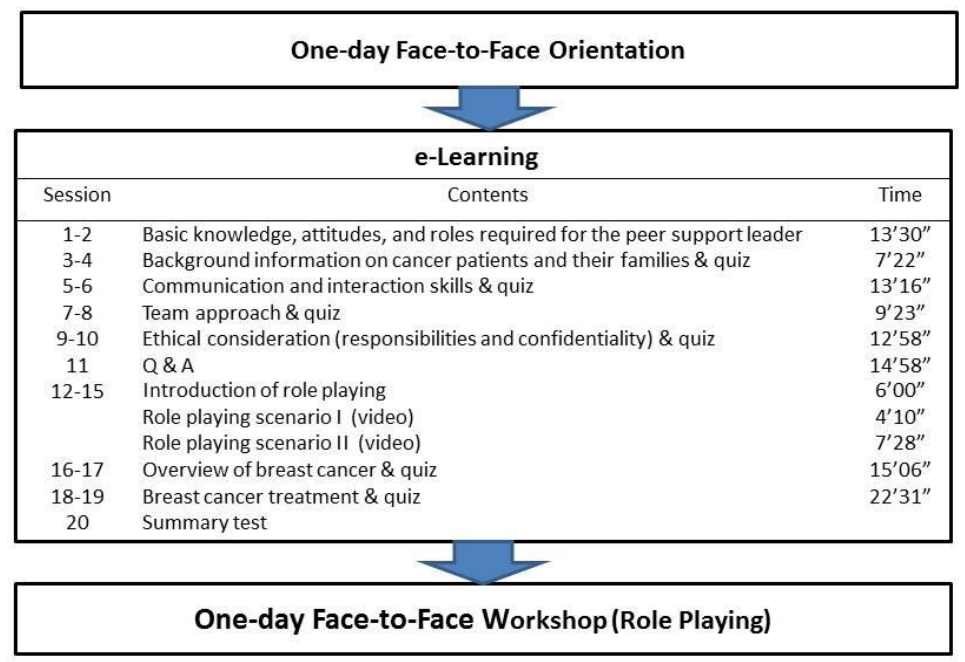

Fig. 1: Blended Learning Model

The e-learning program is provided on the Web. In the e-learning program, the participants learned communication skills (including active listening), interaction skills, ethical considerations (responsibilities and confidentiality), and basic knowledge of breast cancer. There were 20 sessions, and quiz questions were included to allow each participant to self-test their achievement (Fig. 2).

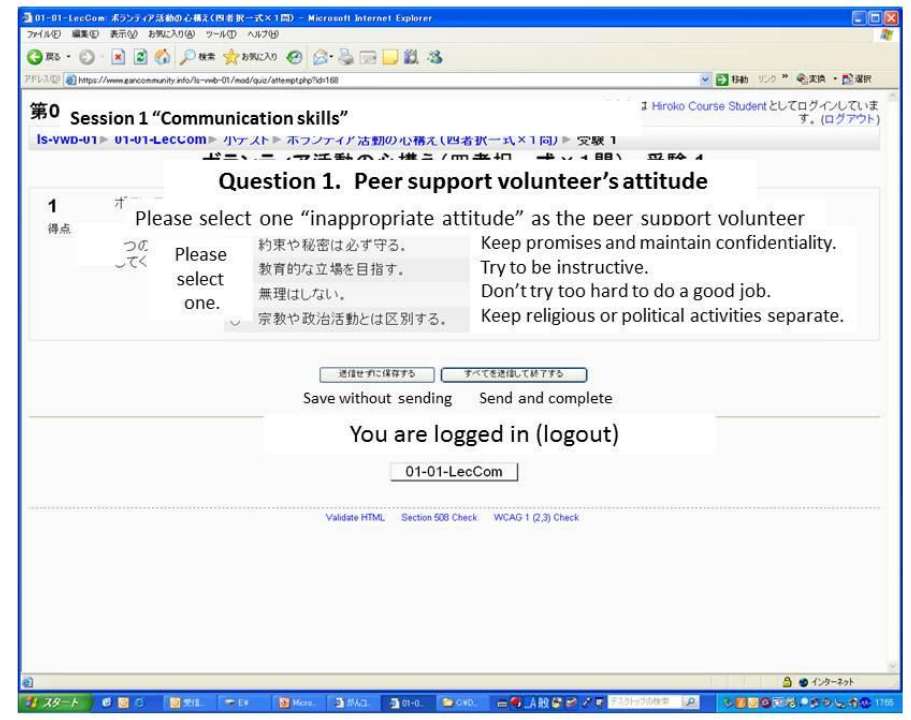

Fig. 2: Quiz on the Website 
The authors developed a handbook for distribution to the peer volunteers. This handbook, describing a step-by-step process of self-learning with basic information on breast cancer and a self-learning record, was used as a reference guide. As the final part of the self-learning program, it was mandatory for the participants to give their comments in an online forum. The comments in the forum were sent to all account holders by e-mail.

After self-learning, the participants attended a one-day workshop and experienced role playing for reinforcement. After guidance about role playing given by nurses, the participants played roles of case patient, support member, and facilitator. The role playing sessions allowed participants to practice handling different cases, and responding to situations. Reflections were drawn from participant discussion.

\subsubsection{Measures}

The feasibility of the peer support training program was determined from recruitment, retention, assessment completion, and usability. We developed a questionnaire to evaluate the usability of the peer support training program. Patient demographics and medical characteristics included age, education, and employment status, treatment modality, and time since cancer diagnosis. The detailed evaluation questionnaire contained rating-type questions with a 5-point Likert scale, ranging from "strongly agree" to "strongly disagree." It also contained open-ended questions that required participants' comments. The participants completed the questionnaire after completing the program. Log-files of the program were analyzed to objectify each participant's pattern of use of the online program (e.g., days to complete the program, the total page view counts) and quiz score.

\subsubsection{Psychosocial outcomes}

Psychosocial outcomes were measured three times over the course of the study: at baseline, immediately after completion of the program, and at the 3 month follow-up. We assessed the participants' perceived quality of life (QOL). The Functional Assessment of Cancer Therapy- Breast (FACT-B) is a diseasespecific evaluation for breast cancer patients with four primary QOL domains (Physical Well-Being, Social/Family Well-Being, Emotional Well-Being, and Functional Well-Being) [19]. The officially validated Japanese version of FACTB [20], a 36-item questionnaire that measures general health-related quality of life 
for any type of cancer (FACT-G) in addition to breast cancer-specific subscales, was used in this study because it was widely used and accessible in Japan.

For depressive symptoms, we used the Center for Epidemiologic Studies Depression (CES-D), which is commonly used to measure symptomatology in cancer patients [21], and the Japanese version of the CES-D was available [22]. The CES-D is a 20-item self-report scale to measure depressive symptoms during the past week. This scale measures depressive symptoms in four domains: depression affect, somatic complaints/activity inhibition, positive affect, and interpersonal difficulties [23].

Since social support factors are related to mental adjustment in cancer patients [24, 25], we used the Japanese version of the Mental Adjustment to Cancer (MAC) scale [24]. MAC is a 40-item self-rating scale with five subscales: fighting spirit, anxious preoccupation, fatalism, helplessness/hopelessness, and avoidance [25].

\subsection{Data Analyses}

Repeated measured one-way analysis of variance (ANOVA) was used to assess adjustment with the psychosocial variables over time, including baseline, postprogram, and 3-month follow-up.

\section{Results}

\subsection{Participants}

Of 28 volunteers with cancer experience agreed to participate in this study, one participant withdrew during the study, and another failed to return the baseline questionnaires despite remainders. A total of 26 women participated in the peer support training program. The rates of retention and completion of the assessments in this study were high, $92.9 \%$ and $89.3 \%$, respectively.

Table 1 and 2 show the demographics and medical characteristics of the participants, respectively (Table $1 \& 2$ ). Their age at baseline ranged from 37 to 75 years (mean 52.3 years; standard deviation [SD] 9.77 years). The mean time since cancer diagnosis was 36.03 months (SD 29.50). 
Table 1: Demographics of the Sample $(n=26)$

\begin{tabular}{lcc}
\hline \multicolumn{1}{c}{ Variable } & $\mathrm{N}$ & $\%$ \\
\hline Age & 3 & 12 \\
$30-39$ & 8 & 31 \\
$40-49$ & 7 & 27 \\
$50-59$ & 6 & 23 \\
$60-69$ & 2 & 8 \\
$70-75$ & & \\
Education & 2 & 8 \\
High school & 5 & 19 \\
Occupational/two-year college & 15 & 58 \\
Bachelor & 2 & 8 \\
Master or above & 2 & 8 \\
Unknown & & \\
Employment status & 13 & 50 \\
Full-time & 4 & 15 \\
Part-time/Temporary & 5 & 19 \\
Housewife & 3 & 12 \\
Retired & 1 & 4 \\
Unemployed & 0 & 0 \\
Other & &
\end{tabular}

Table 2: Clinical Characteristics of the Sample $(n=26)$

\begin{tabular}{lcc}
\hline \multicolumn{1}{c}{ Variable } & $\mathrm{N}$ & $\%$ \\
\hline Treatment modality* & 7 & 27 \\
Mastectomy with reconstruction & 1 & 4 \\
Mastectomy without reconstruction & 20 & 77 \\
Breast conservation & 24 & 92 \\
Hormone therapy & 13 & 50 \\
Chemotherapy & 17 & 65 \\
Adjuvant radiotherapy & 1 & 4 \\
Other & & \\
Time since cancer diagnosis (months) & 6 & 23 \\
6-11 & 8 & 31 \\
12-23 & 1 & 4 \\
24-35 & 2 & 8 \\
36-47 & 3 & 12 \\
48-59 & 6 & 23 \\
$>60$ & & \\
Frequency of PC use & 17 & 65 \\
Almost every day & 4 & 15 \\
3-4 times a week & 3 & 12 \\
1-2 times a week & 1 & 4 \\
\hline
\end{tabular}


Other

1

4

*Multiple answers were allowed for treatment.

\subsection{Usability}

Table 3 shows the participants' responses to questions on the usability of the peer support training program. Overall, positive results on the program were obtained with all questions rated at 4.0 or above. Regarding the usability of the e-learning component, more than $80 \%$ of the participants agreed that the site was easy to navigate, the learning contents were interesting, and the program was easily incorporated into their daily lives. Additionally, $70 \%$ of the participants agreed that the e-learning improved their understanding of role playing, although only $36 \%$ agreed that the online forum was useful.

More than $90 \%$ of the participants agreed that the role playing was useful, and had clear objectives and learning content. Similarly, more than $90 \%$ of the participants agreed that they understood their peer support role from role playing. The participants reported what was effective and what could be improved in the blended learning program. The effects included "key points and methods for communicating with cancer patients were clear," "I learned basic attitudes and approaches to volunteering," "I obtained confidence in my ability to volunteer, and knowledge about activities I have to carry out as a volunteer," while the improvement required included "it would be better if the operation were simplified," "tasks of a shorter duration were preferable" and "real-time opinion exchanges on the website should be utilized."

Table3: Evaluation on the Peer Support Training Program (\%)

\begin{tabular}{lccccc}
\hline \multicolumn{1}{c}{ Survey Question } & $\begin{array}{c}\text { Strongly } \\
\text { Agree }\end{array}$ & $\begin{array}{c}\text { Agree } \\
\text { Neither } \\
\text { Agree } \\
\text { Nor } \\
\text { Disagree }\end{array}$ & $\begin{array}{c}\text { Disagree Strongly } \\
\text { Disagree }\end{array}$ \\
\hline $\begin{array}{l}\text { I . Overall program } \\
\quad \text { The purpose of learning was clear. }\end{array}$ & 65 & 31 & 4 & 0 & 0 \\
$\quad \begin{array}{l}\text { Learning contents were well taught. } \\
\quad\end{array}$ & 35 & 58 & 8 & 0 & 0 \\
$\quad$ Learning contents were interesting. & 62 & 38 & 0 & 0 & 0 \\
$\begin{array}{l}\text { Combining e-learning and face-to-face } \\
\text { sessions was useful. }\end{array}$ & 69 & 31 & 0 & 0 & 0 \\
$\begin{array}{l}\text { Quiz in each session was useful to } \\
\text { understand the learning. }\end{array}$ & 38 & 58 & 4 & 0 & 0
\end{tabular}


$\begin{array}{lllllll}\text { Overall, the quality of the learning } & 38 & 58 & 4 & 0 & 0\end{array}$ experience in this program was satisfactory.

\section{E-learning}

It was easy to navigate the e-learning 38 website.

The e-learning program was easily incorporated into daily life.

The e-learning was interesting.

$\begin{array}{lllll}38 & 54 & 8 & 0 & 0\end{array}$

A considerable effort was needed to continue

the learning program.

The group work was useful.

$\begin{array}{lllll}0 & 8 & 12 & 54 & 27\end{array}$

The online forum was helpful.

$\begin{array}{llll}54 & 23 & 0 & 0\end{array}$

$\begin{array}{lllll}8 & 31 & 35 & 27 & 0\end{array}$

III. Face-to-face sessions

The purposes of the sessions were clear.

$\begin{array}{lllll}54 & 42 & 4 & 0 & 0\end{array}$

The sessions were interesting.

4

$0 \quad 0$

The role playing helped me to understand my volunteer as a volunteer.

The role playing helped me to connect with
the participants.

$50 \quad 46$

$\begin{array}{llll}46 & 4 & 0 & 0\end{array}$

$\begin{array}{lllll}35 & 38 & 27 & 0 & 0\end{array}$

*Values are Likert ratings showing the percentages of particioants responding in each of five levels where $5=$ strongly agree, 4 = agree, $3=$ neither agree nor disagree, 2 = disagree, and $1=$ strongly disagree

\subsection{Psychosocial outcomes}

There were no significant changes in the total scores of CES-D and FACT-B. The anxious preoccupation (AP) score in the MAC scale was significantly declined $(\mathrm{p}=.011)$, while the emotional well-being (EWB) score in FACT-B was significantly improved $(\mathrm{p}=.032)$ (Table 4).

Table 4: Psychosocial Outcomes

$(\mathrm{n}=26)$

\begin{tabular}{lccccc} 
& & & & $(\mathrm{n}=26)$ \\
\hline & mean $(\mathrm{SD})$ & & & & \\
\hline & & $\mathrm{T} 1$ & $\mathrm{~T} 2$ & $\mathrm{~T} 3$ & $F$ \\
CES-D & & $15.88(11.02)$ & $13.31(10.24)$ & $13.31(11.98)$ & 1.656 \\
MAC & & & & &
\end{tabular}




\begin{tabular}{lrrrr} 
Fighting Spirit (FS) & $47.62(7.15)$ & $47.08(6.09)$ & $45.92(9.18)$ & 1.563 \\
Helpless/Hopeless (H) & $9.04(2.41)$ & $8.46(2.90)$ & $9.12(3.34)$ & 1.399 \\
Anxious Preoccupation (AP) & $24.27(4.46)$ & $23.42(4.01)$ & $22.65(3.81)$ & $4.952^{*}$ \\
Fatalism (F) & $16.42(3.09)$ & $16.27(3.37)$ & $16.58(3.13)$ & 0.14 \\
Avoidance (A) & $1.54(0.71)$ & $1.54(0.81)$ & $1.69(0.97)$ & 0.524 \\
FACT-B & & & \\
Physical well-being (PWB) & $20.46(6.27)$ & $21.35(5.85)$ & $21.69(5.48)$ & 1.655 \\
Social/family well-being (SWB) & $18.56(7.04)$ & $19.25(6.55)$ & $18.73(6.41)$ & 0.29 \\
Emotional well-being (EWB) & $15.23(6.80)$ & $17.04(4.56)$ & $16.96(4.43)$ & $3.688^{*}$ \\
Functional well-being (FWB) & $20.12(5.29)$ & $20.73(4.49)$ & $21.38(5.34)$ & 1.605 \\
Breast cancer subscale (BCS) & $21.77(5.58)$ & $22.31(5.71)$ & $21.93(4.92)$ & 0.369 \\
Trial outcome index (TOI) & $62.35(14.59)$ & $64.38(13.80)$ & $65.0(12.92)$ & 1.847 \\
FACT-G total score & $74.37(20.45)$ & $78.37(17.15)$ & $78.77(16.45)$ & 2.67 \\
FACT-B total score & $96.13(24.62)$ & 100.67 & 100.69 & 2.555 \\
& & $(21.86)$ & $(19.92)$ & \\
\hline
\end{tabular}

$* \mathrm{p}<0.05$

\subsection{Log files}

The time taken to complete the total of 20 sessions in the e-learning program ranged from 0.16 to 57.80 days (mean 18.69; SD 10.98). The total page view counts (number of clicks on the Web pages) showing the participants' activity ranged from 107 to 400 (mean 229, SD 78) (Fig. 3). The participants were the most active from 20:00 to 20:59, accounting for $15 \%$ of the total activity in one hour. It was quiet from midnight to early morning, while the activities were distributed from 9:00 to 22:00. All participants answered the quiz correctly. 


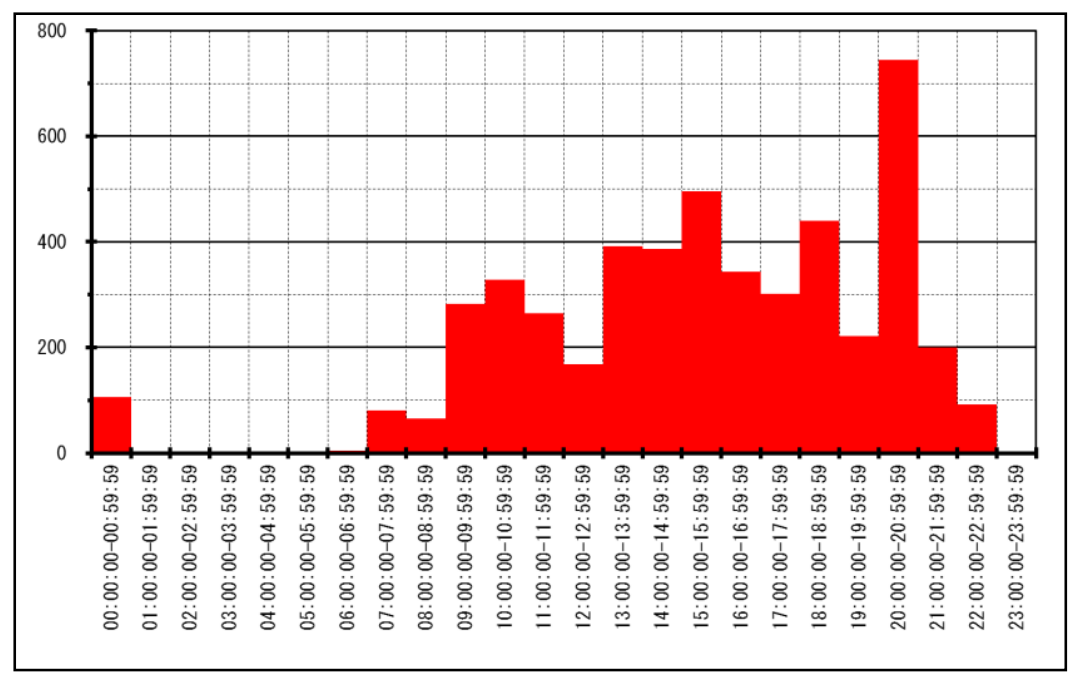

Fig. 3: Hourly Total Page Views

\section{Discussion}

The pilot study demonstrates that the blended learning model used in this peer support training program is feasible. With successful recruitment, the rates of retention and completion of the assessments in this study were high.

The sample characteristics might have affected these success rates. All of the participants had attended more than two breast support groups and thus they recognized the importance of peer support, resulting in very few dropouts during the study period. Furthermore, 67\% of the participants had high educational backgrounds (bachelor's degree or above) and frequently used a personal computer, indicating a population that was well educated with good computer literacy. This background facilitated participation. Also, the favorable ratings of the program may have contributed to the participant retention and assessment completion in this study.

The usability of the program was highly rated. In particular, $70 \%$ of the participants in this study agreed that e-learning helped them to understand the role playing. Role playing is challenging for participants, and may cause anxiety [26]. In this program, the participants experienced online modular training before actual role playing and group discussion. The virtual role playing mirrors participant interactions and common problems they face in an actual support group, therefore participants can be prepared for actual situations. Then, role playing in face-toface sessions reinforce what they have learned in e-learning, and future leaders experience how to manage group dynamics, and learn facilitation skills.

In contrast, only $36 \%$ agreed that the online forum was useful. This was because the forum was held at the end of the program. The results suggest that the 
online forum would be more effective if the participants could communicate with their peers during the self-learning period rather than only the end of the program.

Group leader stress and burnout are often reported, and even significantly related to the closure of the group [13]. A high burden on the group leader should be prevented. This peer support training program did not negatively affect their psychosocial states of the participants. Their CES-D and FACT-B scores were not significantly changed. The anxious preoccupation (AP) score in the MAC significantly declined during the study period. Moreover, the emotional wellbeing (EWB) score in FACT-B was significantly improved, suggesting the positive effects of the program on the peer support volunteers. This study, however, did not examine the effectiveness of the program. A future study examining the program's effectiveness is planned.

The average duration required to complete the e-learning program was 19 days, ranging from 0.16 to 57.80 (SD 10.98). The participants had to balance work and family in addition to continuing treatment, and only had limited time available for learning, but accessing the content of the program at their own pace via the Internet made it easier for them to continue the program. They were most active on the site between 20:00 - 22:59 at night, accounting for 15\% of the total page views. More activity on the site was seen in the afternoon than in the morning, while the activity was low during meal times. Because the participants were women, they may have been busy preparing meals.

A large British survey reports that there are few differences between professionally-led and peer-led support groups [14]. Given the increasing number of survivors and limited availability of health care professionals, more peer-led support groups will be needed. This blended learning training program is useful to increase peer support groups led by formally trained leaders. Also, these findings provide information to help guide the design of future peer support training programs in terms of days required for the program and best times to hold specific activities. For example, the most suitable time period in which to provide mentoring or tutoring services using the chat function to boost peer exchange is 20:00-20:59. Training programs should cover necessary topics but clearly define peer support roles, and avoid overloading the participants.

\section{Limitations}

There was no control group in this study, because its aim was to test the initial feasibility of the program. Feasibility studies may not themselves be randomized and do not evaluate the effectiveness of the program [27]. The sample of this study may be biased because most of the participants were highly educated and had relatively high computer literacy. Future research should include a randomized controlled trial (RCT) with a larger sample to examine the effectiveness of the peer support training program. 


\section{Conclusion}

This study demonstrates that the peer support training program using a blended learning model is feasible and practical. This blended learning model helps participants easily access the program through the advantage of e-leaning and reinforces their learning with traditional face-to-face sessions including role playing. This is a potentially low-cost approach to reducing regional differences in training peer support volunteers. Additional research is needed to determine the effects of the training program in RCT.

\section{Acknowledgments}

The authors would like to thank Takahiro Niiya for his contribution in developing the e-learning program, as well as our colleagues at St. Luke's International Hospital Breast Cancer Center for their valuable comments on the learning contents. This study was funded by the Ministry of Education, Sports, and Science Technology of Japan, Scientific Research (A) No. 19209066.

\section{Conflicts of interest}

All authors declare they have no conflict of interest.

\section{References}

[1] N. Doyle, Cancer survivorship: evolutionary concept analysis, J Adv Nurs 62, 4 (2008), 499-509.

[2] M. Carey, S. Lambert, R. Smits et al, The unfulfilled promise: a systematic review of interventions to reduce the unmet supportive care needs of cancer patients, Support Care Cancer 20 (2012), 207-219.

[3] A. Montazeri, Health-related quality of life in breast cancer patients: A bibliographic review of the literature from 1974 to 2007, J Exp Clin Cancer Res 27 (2008), 32.

[4] PJ. Goodwin, M. Leszcz, M Ennis et al, The effect of group psychosocial support on survival in metastatic breast cancer, New Engl J Med 345, 24 (2001), 1719-1726.

[5] J. Giese-Davis, C. Bliss-Isberg, K. Carson et al, Brief Repost: The effect of peer counseling on quality of life following diagnosis of breast carcinoma: An observational study, Psychooncology 15, 11 (2006), 1014-1022.

[6] M. L. Kwan, I. J. Ergas, C. P. Somkin et al, Quality of life among women recently diagnosed with invasive breast cancer: the Pathways Study, Breast Cancer Res Treat 123, 2, (2010), 507-524. 
[7] M. Epplein, Y. Zheng, W, Zheng et al, Quality of life after breast cancer diagnosis and survival, J Clin Oncol 29, 4 (2011), 406-412.

[8] R. E. Gray, M. Fitch, C. Davis et al, A qualitative study of breast cancer selfhelp groups Psychooncology 6 (1997), 279-289.

[9] L. M. Hoey, S. C. Ieropoli, V. M. White et al, Systematic review of peersupport programs for people with cancer, Patient Educ Couns 70, 3 (2008), 315-337.

[10] J. Usshera, L. Kirstena, P. Butowb, M. Sandovala, What do cancer support groups provide which other supportive relationships do not? The experience of peer support groups for people with cancer, Social Science \& Medicine 62, (2006) 2565-2576.

[11] P. Arnstein, M. Vidal, C. Wells-Federman et al, From chronic pain patient to peer: benefits and risks of volunteering, Pain Manag Nurs 3 (2002), 94-103.

[12] [12] M. Price, B. Phyllis, L. Kirsten, Support and training needs of cancer support group leaders: a review, Psycho-Oncology 15 (2006), 651-663.

[13] P. Butow, D. MPH, J. Ussher, Sustaining Leaders of Cancer Support Groups: The Role, Needs, and Difficulties of Leaders, Social Work in Health Care 42, 2, (2005).

[14] C. Stevinson, A. Lydon, Z. Amir, Characteristics of professionally-led and peer-led cancer support groups in the United Kingdom, J Cancer Surviv 4 (2010), 31-338.

[15] Ministry of Health, Labor and Welfare (2007) Cancer Control Act. http://www.mhlw.go.jp/english/wp/wp-hw2/part2/p3_0026.pdf. Accessed January 12, 2012.

[16] Ministry of Health, Labor and Welfare (2010) Interim Report of Cancer Control Act. http://www.mhlw.go.jp/bunya/kenkou/gan_keikaku.html. Accessed January 31, 2012.

[17] S. W. Chen, J. Stocker, R. H. Wang et al. Evaluation of self-regulatory online learning in a blended course for post-registration nursing students in Taiwan, Nurse Educ Today 29, 7 (2009), 704-709.

[18] D. A. Cook, Internet-Based learning in the Health Professions A metaanalysis, JAMA 300, 10 (2008), 1181-1196.

[19] M. J. Brady, D. F. Cella, F. Mo et al, Reliability and validity of the Functional Assessment of Cancer Therapy-Breast quality-of-life instrument, J Clin Oncol 15, 3 (1997), 974-986.

[20] K. Shimozuma, N. Eguchi, Development and clinical application of QOL measurement instruments for cancer patients (I), 2001 - Development of the Japanese version of QOL questionnaires developed in the West and validation of reliability and validity of "FACT-B," a QOL questionnaire for breast cancer patients (in Japanese) http://www.jmari.med.or.jp/research/dl.php?no=47. Accessed January 18, 2012. 
[21] S. Oba, N, Takatsuka, C. Nagata et al, Causal attributions to epidemiological risk factors and their associations to later psychological adjustment among Japanese breast cancer patients, Support Care Cancer 17 (2009), 3-9.

[22] S. Shima, T. Shikano, T. Kitamura, A new self-report depression scale, Psychiatry 27, 1985, 717-723 (in Japanese).

[23] L. S. Radloff, The CES-D scale: a self-report depression scale for research in the general population, Appl Psychol Measurement 1, 3 (1977), 385-401.

[24] T. Akechi, M. Fukue-Saeki, A. Kugaya et al, Psychometric properties of the Japanese version of the Mental Adjustment to Cancer (MAC) scale, Psychooncology 9, 2000, 395-401.

[25] M. Watson, S. Greer, J. Young et al, Development of a questionnaire measure of adjustment to cancer: the MAC scale, Psychol Med 18 (1988), 203-209.

[26] N. A. Kinnane, T. Waters, S. Aranda, Evaluation of a pilot 'peer support' training programme for volunterrs in a hospital-based cancer information and support center. Support Care Cancer 19, 2011, 81-90.

[27] M. Abrain, M. Abrain, M. J. Campbell, C. L. Cooper et al, What is a pilot or feasibility study? A review of current practice and editorial policy, BMC Med Res Methodol 10 (2010), 67. 